\title{
Approach for Analysis and Improved Usage of Digital Cultural Assets for Learning Purposes
}

\section{Paneva-Marinova, R. Pavlov, N. Kotuzov}

Institute of Mathematics and Informatics, Bulgarian Academy of Sciences, 1113 Sofia, Bulgaria

E-mails: dessi@cc.bas.bgradko@cc.bas.bg nikolay.kotuzov@bultreebank.org

Abstract: This paper presents an approach for analysis and improved usage of digital cultural assets for non-formal learning purposes in digital culture ecosystems. The digital culture ecosystems, their features, and an exemplar are discussed.

Keywords: Digital culture ecosystem, analysis, improved usage, digital cultural assets, non-formal learning.

\section{Introduction}

In recent years, the research community has demonstrated considerable interest in art and culture and their digitalization, online presentation and wide dissemination. However, knowing and understanding artworks is not an easy task and requires flexible learning methods, especially when they will be used online. Current education strategies for learning digital art and culture point to the investigation and deployment of workable learning approaches for better understanding, creative thinking, learning-by-doing and learning-by-authoring, engaging users in a more active participation during the knowledge-perceiving phase [21]. Some of the learning purposes are:

- observing, analysing and studying target cultural domains;

- finding or verifying trends in the cultural domain, finding new trends, influences, and problems, reach new results;

- making new projects for shared and/or linked Digital Cultural Assets (DCAs), new art or learning projects, documentaries, performance;

- content-dependent use of DCAs for different purposes, incl. interactive virtual exhibitions, gaming and gamification, storytelling, mobile applications, studying, etc.

- stimulation of new research and promotion of innovative usage and user interaction with the cultural heritage, etc.

A key assumption is that the improved use and observation of knowledge, related to cultural and historical heritage will help addressing some of the problems 
with handling large volumes of digital cultural data and objects, better cultural domain understanding as well as beneficial user interaction with DCAs [22]. Current problems may be overcome, e.g., poorly adaptive and customizable presentation of objects, difficulties in context-based access and use, lack of uniform interpretation, insufficient attractiveness of presentation, lack of unified access to many different digital repositories of cultural and historical heritage, etc.

This paper discusses digital culture ecosystems and the processes of content aggregation, observation, and their study, as well as the users' roles and activities in the mentioned context. It presents an approach for analysis and improved usage of digital cultural assets for non-formal learning purposes.

\section{Digital culture ecosystems. Exemplar}

The paradigm of ecosystems for digital cultural assets [23] (also called Digital Cultural Ecosystems, DCEs) appears to respond to the growing willingness to share the wealth of cultural resources and continuous research and study of cultural treasures. These systems virtually assemble various digital collections, archives, virtual museums, digital libraries and cultural heritage sites in order to facilitate the access to their resources, bringing cultural content to new audiences in novel ways [23].

The European activities in this field are promoted and supported by the Horizon 2020 programme of EC H2020-EU.3.6.3. - Reflective societies - cultural heritage and European identity in the priority REFLECTIVE-6-2015 - Innovation ecosystems of digital cultural assets. The following research and innovation projects are successfully evaluated and started in 2016: I-Media-Cities - Innovative e-Environment for Research on Cities and the Media [11], ARCHES - Accessible Resources for Cultural Heritage EcoSystems [5], CROSSCULT - Empowering Reuse of Digital Cultural Heritage in Context-aware Crosscuts of European History [7], and ArchAIDE - Archaeological Automatic Interpretation and Documentation of cEramics [4]. Activities under these projects aim to:

- "stimulate new research perspectives for the humanities and social science communities; promote further the use of digital cultural heritage allowing its reinterpretation towards the development of a new shared culture in Europe.

- provide innovative and creative methods for approaching cultural assets and for generating applications and services to access and exploit the rich and diverse European digital cultural heritage in a sustainable way.

- foster collaboration between those with primary expertise in the interpretation of cultural data and researchers with complementary expertise in digital and interactive frameworks" [23].

I-Media-Cities [11] is an initiative of 9 European film libraries, 5 research institutions, 2 technology providers and a specialist in digital business models to share access to and valorise audio-visual content from their collections for research and creative purposes in a wide range of social sciences (sociology, anthropology, urban planning, etc.). 
ARCHES [5] aims to create more inclusive cultural environments, particularly for those with difficulties and disabilities associated with perception, memory, cognition and communication. This goal will be achieved through in-depth research analysis and the development of innovative applications, functionalities and experiences based on the reuse and re-development of the digital resources. Project research methodology situates real user needs at the heart of an iterative design and implementation process, through the active involvement of the users in three pilot exercises.

CROSSCULT [7] aims to make reflective history a reality in the European cultural context, by enabling the re-interpretation of European histories through cross-border interconnections between cultural digital resources, citizen viewpoints and physical venues. The project has two main goals. The first goal is to lower EU cultural barriers and create unique cross-border perspectives, by connecting existing digital historical resources and by creating new ones through the participation of the public. The second goal is to provide long-lasting experiences of social learning and entertainment that will help towards the better understanding and re-interpretation of European history.

\subsection{Digital cultural ecosystem features}

In nature, an ecosystem is an area where organisms interact with one other as well as with the non-living parts of the environment. In the digital cultural ecosystem, various "digital organisms" (viz. collections, archives, virtual museums, digital libraries, cultural heritage site, etc.) also interact with one another as well as with the living part of the environment (viz. users). Formally, a digital cultural ecosystem can be huge, covering joint content management systems of one country or a region (similarly to a large forest or lake in the nature), but it can also be small, such as a virtual museum or a private collection of artefacts (the nature analogues: a puddle of water or only a tree). "Digital organisms" "work" through services and tools to satisfy their users. DCEs aggregate heterogeneous resources leaning on interoperability support of its building blocks (in [18] authors have proposed a solution for content interoperability between various digital libraries).

In the CultEcoSys project [8] we perceive Digital Libraries (DLs), virtual museum, cultural website, etc., as small ecosystems for digital cultural assets. For example, current cultural heritage DLs demonstrate wide range of applicable services and tools for re-using and repurposing digital assets (or objects, DCOs), paving the way for wider exploitation of cultural resources and boosting innovation. Fig. 1 and Fig. 2 depict the DL content flow, the main content units and the user's activities for manipulation in DL. 


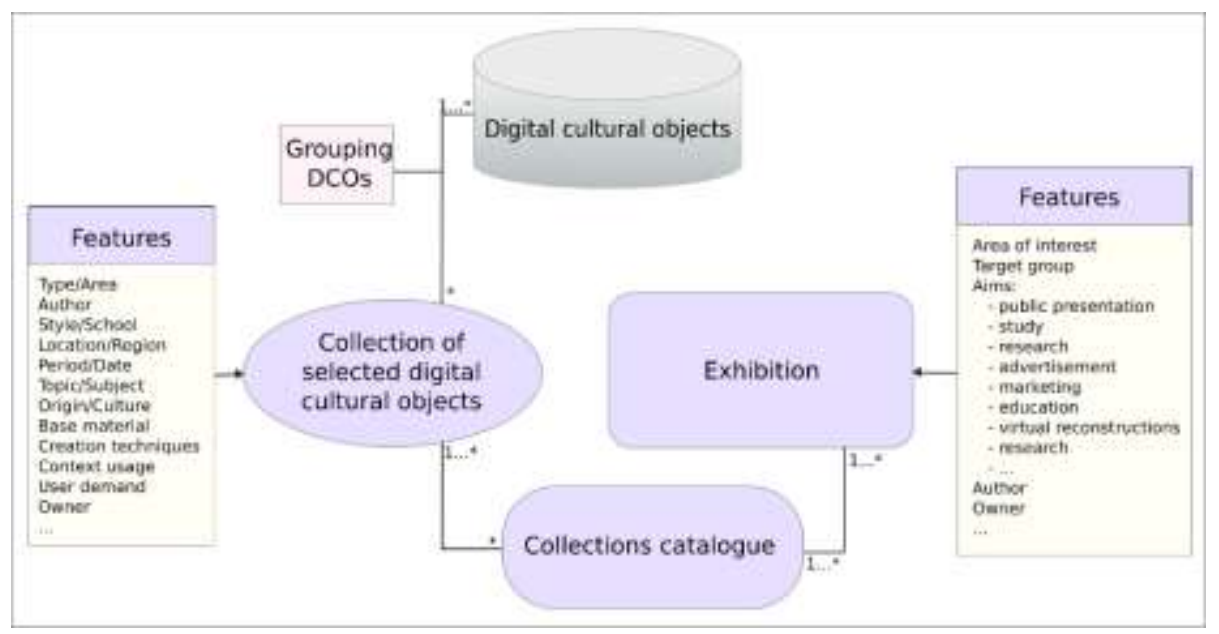

Fig. 1. Content flow in a digital library

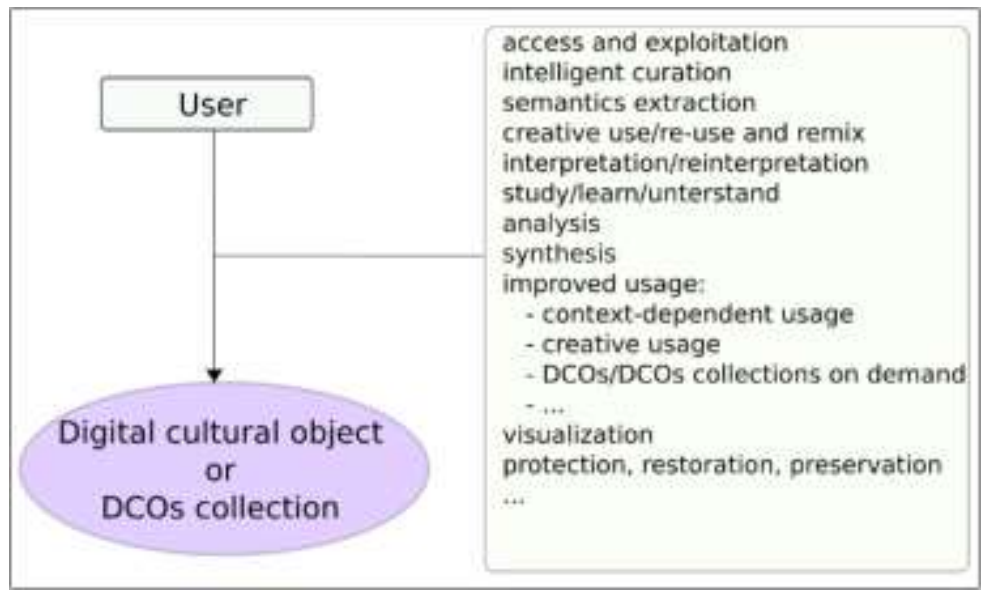

Fig. 2. Main content units and activities for their manipulation in a digital library

Digital cultural objects are the smallest content units in the DCE. Contextbased grouping of DCOs creates a collection with a wide variety of usage. The DCOs could be selected according to their type and area, author, style, school, location, date, subject, origin, context of usage, etc. The collections are saved in catalogues for further inclusion and display in exhibitions.

In general, the user's manipulations with DCOs and DCOs collections are related to access and exploitation, curation, semantic extraction, use/re-use and remix, analysis, study, etc. [19].

The main content units and the activities for their manipulation can be extended according to the concrete DCE' aims, marketing and advertising strategies, target groups, etc. In this study, we concentrated on models and visions for improved use, research and delivery of digital cultural resources in DCE. 


\section{Content analytics in a digital cultural ecosystem}

The long-term observation of the user's needs in DLs gives us the idea to look at analysis techniques and tools for improved usage of digital cultural assets [7, 9, 10, $16,19]$ in DCEs, incl. new social usage, personalised usage, re-usage. The analysis includes a specific research/study about a particular object or a whole domain, and aims to collect useful information about their properties, characteristics, and integral parts. The main purpose is finding different characteristics about the objects or domains being analysed, their structure, dependencies, internal and external relationships between their constituents, etc. Those are revealed by:

- determining the main trends in the development in the domain as well as the direction and dynamics of the development;

- discovering the key factors and conditions that directly affect the changes and developments in the domain; examining the degree of their influence and the direction of the changes;

- determining the degree of influence of the domain upon other related domains;

- identifying new trends in the development of the analysed domain;

- defining current problems, boundaries and limitations in the development of the analysed domain, as well as problems related to them and possible areas of improvement;

- evaluating the results in the domain's development.

The main users of the learning analysis method in the DCEs are learners and educators, but there could also be researchers/professionals, connoisseurs or tourists.

To implement the analysis method (mainly for learning purposes), the practical research/learning problem is divided into series of steps, leading to its solution. The steps are defined as a formula, which combines actions (they are based on the Bloom Taxonomy [6] and its "recommended vocabulary" of actions (called skills by Bloom), leading to results of the learning process (learning objective) in a certain area [20]) with content units [17]. For example, the user, (i.e., learner) classifies digital art objects (paintings) accordingly to their author, i.e., he performs an action of grouping objects based on certain criteria. The selection could be further arranged according to the time feature and the user can see/discover the main trends, direction and dynamics in the authors' artwork.

Furthermore, the following data analysis practices often use qualitative methods. The analysis process involves three activities essentially [13]: 1) data reduction: the process of selecting, focusing, simplifying, abstracting, and transforming raw data; 2) data display: the assembling of information in an organized way; and 3) conclusion drawing and verification: the observation of regularities, patterns, explanations, possible configurations, causal flaws, and propositions that are verified and tested for their plausibility and validity.

Fig. 3 depicts analysis actions provided to the users (viz. viewers: learner, educator, researcher/professional, tourist, and connoisseur; editor; moderator; administrator) of the digital cultural ecosystem. The analysis actions are separated 
into two types: Standard analysis and Specific analysis. As shown below, the standard analysis actions include the basic functionality for objects/collections search, review, selection and management. The specific analysis actions aim to improve the manipulation activities with DCE assets. We regard the following DCE content types:

1. Digital Cultural Object (DCO);

2. DCOs collection (public DCOs collection, private DCOs collection, temporary DCOs group, etc.);

3. DCOs exhibition;

4. DCOs presentation;

5. DCOs learning project.

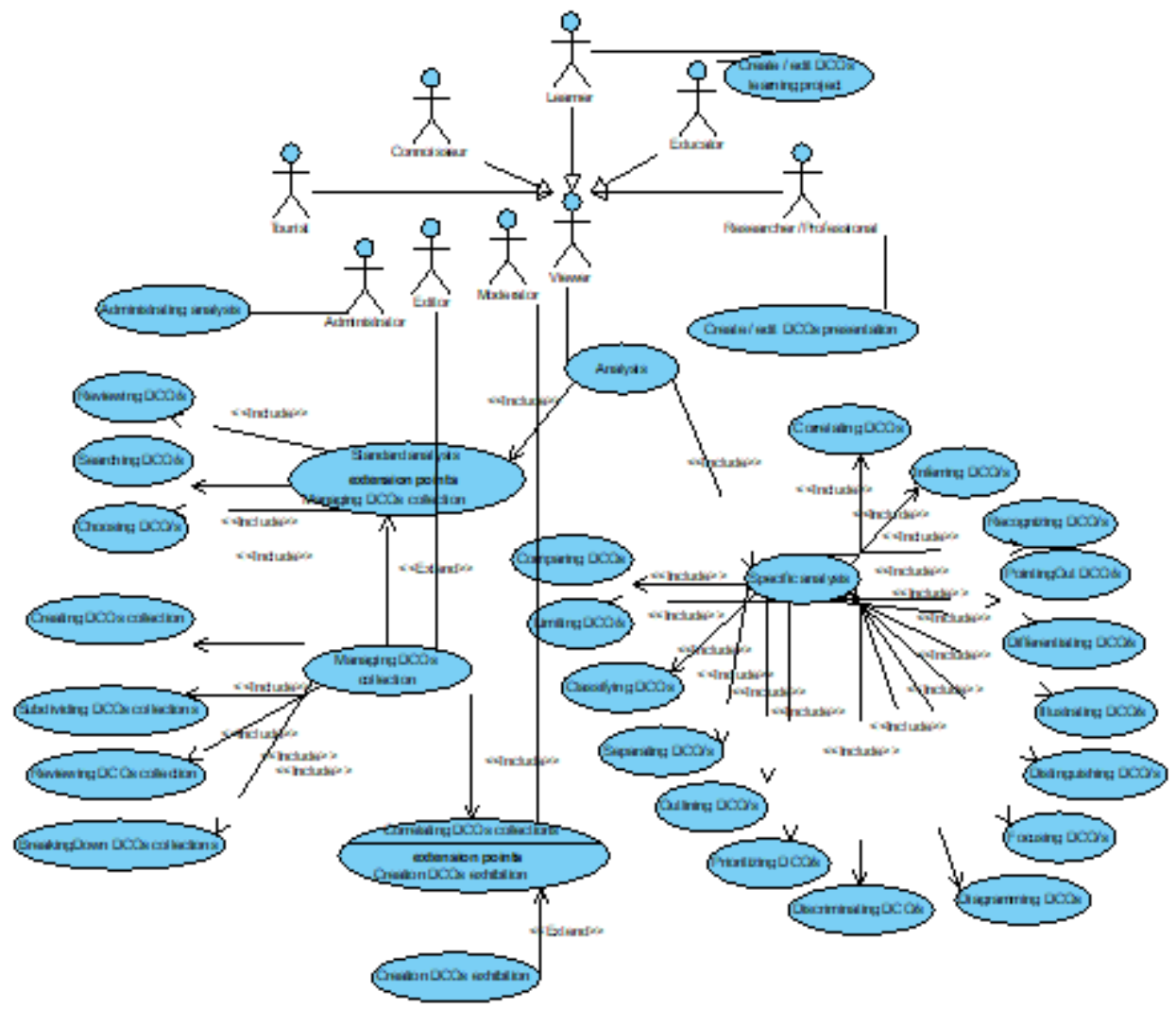

Fig. 3. Analysis actions provided to the DCE users

Following data analysis sourcebook Miles, Huberman and Saldana [13], the analysis actions from Fig. 3 could be grouped by their similarity and feasibility (see Table 1). 
Table 1. Analysis actions according to data reduction, data display and conclusion drawing and verification

\begin{tabular}{|c|c|c|}
\hline Data reduction & Data display & $\begin{array}{l}\text { Conclusion drawing } \\
\text { and verification }\end{array}$ \\
\hline $\begin{array}{l}\text { Searching DCO/s } \\
\text { Choosing DCO/s } \\
\text { Correlating DCOs } \\
\text { PointingOut DCO/s } \\
\text { Distinguishing DCO/s } \\
\text { Prioritizing DCO/s } \\
\text { Focusing DCO/s } \\
\text { Recognizing DCO/s }\end{array}$ & $\begin{array}{l}\text { Reviewing DCO/s } \\
\text { Reviewing DCOs collection } \\
\text { Creating DCOs collection } \\
\text { Correlating DCOs collections } \\
\text { Differentiating DCO/s } \\
\text { Limiting DCO/s } \\
\text { Separating DCO/s } \\
\text { Discriminating DCO/s } \\
\text { Subdividing DCOs collections } \\
\text { BreakingDown DCOs collections } \\
\text { Creating DCOs presentation } \\
\text { Creating DCOs learning project }\end{array}$ & $\begin{array}{l}\text { Outlining DCO/s } \\
\text { Illustrating DCO/s } \\
\text { Diagramming DCOs } \\
\text { Inferring DCO/s }\end{array}$ \\
\hline
\end{tabular}

Moreover, the different needs of the DCE actors have to be considered. For example, the learners and educators demand for essential information and services for developing collections in line with pedagogical requirements, viz. the creation of DCOs learning projects or learning context-dependant DCOs presentations. Other actors could have different needs.

The next scenario demonstrates a real usage of the DCE analysis actions by professionals - the artists.

The analysis actions (or part of them) will assist, for example, the artists in their preliminary preparation (research, analysis) that they make before creating a new piece of artwork. DCE will give them possibilities for review and analysis of digital copies of artworks in a specific domain, topic, etc., also displaying additional descriptive information (metadata) about them (if available).

In particular, let the artist (viz. an iconographer) plan to paint a specific theme/character - "The Virgin" $\rightarrow$ General goal.

In the DCE environment, he/she performs a search by the specified theme/character and review of the found DCOs $\rightarrow$ Task 1 and Task 2.

The next step is the additional choice of a specific subset of the main theme, for example, "The Virgin Hodegetria" $\rightarrow$ Task 3.

The selected objects are separated/arranged according to their creation time/period, which brings different characteristics about the objects from iconographic and technological perspective/point of view $\rightarrow$ Task 4 .

After that, the system could distinguish DCOs by a selected school, point out $\mathrm{DCO} / \mathrm{s}$, including components for new artwork and prioritize one or several of them as a main sample/s $\rightarrow$ Task 5 and Task 6.

The final step is the inferring phase, which aim is to consider and present a conceptual solution/project of the new artwork, using the selected DCOs and their components $\rightarrow$ Task 7 .

Current solutions for improved access to the DCEs content and its effective usage are very restricted and mainly cover the basic analysis actions, mentioned above. The idea for creative and satisfying user experience in the context of advanced content manipulation is realized only by extended search. DCEs do not 
even provide a minimal content analysis functionality. When the user is a learner/researcher, or has learning purposes in the environment (which is very common), such "one size fits all" solutions are not enough to satisfy his/her needs [2]. The reasons for this gap are the low personalization and "real-time" integration of content according to the users' interests and needs in these systems, non-effective content exploitation, and the missing context-based usage of the resources.

Some initial experiments with the presented learning analysis are done during the SINUS project [24] and discussed in [1]. Current and future research work will concentrate on the improvement of the presented learning analysis issues during the ongoing project "Concepts and Models for Innovation Ecosystems of Digital Cultural Assets". The aim of the project is to develop optimal and feasible conceptual models and methods of analysis, understanding, interpretation, contextdependent use and sharing of content in ecosystems for digital culture in new ways and through innovative means for fuller delivery of knowledge to digital collections and archives of cultural artefacts [8].

\section{Towards "real-time" integration of DCOs according the DCE viewers' needs}

In DCEs, like in other software applications, the usability is a measure of product use whereby users achieve concrete objectives in varying degree of effectiveness, efficiency and satisfaction, within a specific context of use [12]. However, when we think about the user and his/her experience we have to consider the following facets of the user experience [14]: useful, usable, findable, credible, desirable, accessible and valuable. In our study, we propose the "real-time" integration of the DCE content units according the users' needs as a means for improved user experience.

"Real-time" integration of the DCE content according the user's needs could be defined as an automatic content aggregation based on the users' specific requirements, in particular, the viewer. The user and respectively his/her needs, interests, goals and preferences have to be carefully studied and have to become the starting point for DCE functionality development. Moreover, we need to know:

- What are the user's motivations for exploring the target DCE? What is the force that drives the user to be engaged in observation activities?

- What is the user's previous experience that may have an impact?

- What the user really does in the DLE? How is something done or accomplished?

- How does the user define his/her own needs, etc.

This "real-time" content integration could be defined also as a type of personalization, covering:

- "Selection and recommendation of information resources according to the personal characteristics of the user on one hand and according to the user's behaviour in the environment on the other;

- Adaption of the user interface, the means of navigation, the display format and the ways of providing information resources" [16]. 
Some formal logics linking certain user's actions (or user profile) with a certain system response (single action or series of actions), delivering selected $\mathrm{DCO} / \mathrm{s}$, follow bellow.

For example, let:

$A=\bigcup_{i=1}^{l} A_{i}$ be the taxonomy of the adaptation aims (for example, content adaptation, interface adaptation, etc.), where $l$ is the overall number of adaptation aims;

$B=\bigcup_{i=1}^{s} B_{i}$ be the taxonomy of the adaptation ways (for example, showing explanations for object/s, showing a selection of objects/parts of the objects, showing groups of objects according to some criteria, etc.), where $s$ is the overall number of adaptation ways;

$$
X=\bigcup_{i=1}^{n} X_{i} \text { be the taxonomy of user model characteristics, where } n \text { is the }
$$
overall number of user model characteristics $[15,16]$;

$Y=\bigcup_{i=1}^{k} Y_{i}$ be the taxonomy of the DCE objects, where $k$ is the overall number of the DCE objects;

$Z=\bigcup_{i=1}^{m} Z_{i}$ be the taxonomy of the user's actions in the DCE environment (reviewing, choosing, etc.), where $m$ is the overall number of the user's actions;

$$
D=\bigcup_{i=1}^{p} D_{i} \text { be the taxonomy of the devices used by the user to access the DCE }
$$
environment (PDAs, PCs, smart phones, etc.), where $p$ is the overall number of the devices.

Then:

$$
\begin{gathered}
\forall p, k, j, r, l, m, \forall a_{i p} \in A_{p}, b_{i k} \in B_{k}, x_{i j} \in X_{j}, y_{i r} \in Y_{r}, z_{i l} \in Z_{l}, d_{i m} \in D_{m} \\
\exists g_{i}, g_{i} \in G: a_{i p} \cup b_{i k} \cup x_{i j} \cup y_{i r} \cup z_{i l} \cup d_{i m} \rightarrow g_{i},
\end{gathered}
$$

i.e., the association of elements of $A, B, X, Y, Z$ and $D$ the following axiom may be used:

$$
\begin{aligned}
& \operatorname{IF}\left(A_{1}=a_{1 p}, A_{2}=a_{2 p}, \ldots, A_{i}=a_{i p}, \ldots\right), \\
& \quad \operatorname{AND}\left(B_{1}=b_{1 k}, B_{2}=b_{2 k}, \ldots, B_{i}=b_{i k}, \ldots\right), \\
& \operatorname{AND}\left(X_{1}=x_{1 j}, X_{2}=x_{2 j}, \ldots, X_{i j}, \ldots\right), \\
& \operatorname{AND}\left(Y_{1}=y_{1 r}, Y_{2}=y_{2 r}, \ldots, Y_{i}=y_{i r}, \ldots\right), \\
& \operatorname{AND}\left(Z_{1}=z_{1 l}, Z_{2}=z_{2 l}, \ldots, Z_{i}=z_{i l}, \ldots\right), \\
& \operatorname{AND}\left(D_{1}=d_{1 m}, D_{2}=d_{2 m}, \ldots, D_{i}=d_{i m}, \ldots\right),
\end{aligned}
$$


$\operatorname{THEN}\left(G=g_{1}, g_{2}, \ldots, g_{n}\right)$,

where $G$ is a selection of sets of objects (and/or part of them) from the DCE knowledge domain $(G \supseteq Y$ ) that are adapted according to concrete adaptation aims and ways and are consistent with the user model, the user's actions, and access devices.

For example:

IF ( $A_{1}=$ Adaptation aim: content adaptation),

AND ( $B_{1}=$ Adaptation way: display of definite descriptive characteristics of the DCO, descriptive characteristics: $h_{1}, h_{2}, \ldots, h_{n}$ ),

AND ( $X_{1}=$ Knowledge level in the DCE domain: Advanced, Interest: Artwork),

AND ( $Y_{1}=$ Art objects: Sculptures, $Y_{2}=$ School/Style: Renaissance),

$\operatorname{AND}\left(Z_{1}=\right.$ User action: Reviewing DCOs),

THEN ( $G=$ Selection of DCOs: Renaissance sculptures (artworks) with descriptive characteristics $h_{1}, h_{2}, \ldots, h_{n}$, presented for advanced level review).

We could also consider cases that are more complex where the DCE engine extracts the user model characteristics automatically, i.e., we have dynamically generated a user model, or the user requests more than one action at once and the system maintains various adaptations in addition. Moreover, if DCE developers pursue the support of full semantic interconnection between DCOs, the semantic inferring needs to be the core issue.

\section{Conclusions}

The great fragmentation of the major historical and cultural sources related to history and culture in various digital collections, libraries and repositories puts on the agenda the question of providing users with opportunities for their joint consideration and study in order to fully utilise all semantic interconnection between them, overriding physical distance and the specifics of the digital storage of each source. Furthermore, the volume of digital cultural objects and data is growing rapidly. One possible approach to solving this problem is linked to the creation of context-dependent models for improved use, research, and analysis of large volumes of digital cultural resources and support of "real-time" integration of DCOs according the users' needs [2]. We presented an approach for analysis and improved usage of digital cultural assets as a base for supporting personalized observation and improved knowledge perception, using analysis actions, suitable for non-formal learning processes.

The results from this study are actually steps in the realization of a research project "Concepts and Models for Innovation Ecosystems of Digital Cultural Assets" [8], which is already mentioned above. The conclusions made from the research will be used for defining the directions for the real implementation of the proposed approach, including possible transformations of the analysis actions set, 
tuning the algorithms and their parameters, etc., in order to achieve more accurate results and to provide more useful DCE assets to the users.

Acknowledgements: This work is partly funded by the Bulgarian NSF under the research project No DN02/06/15.12.2016 "Concepts and Models for Innovation Ecosystems of Digital Cultural Assets", WP2 - Creating models and tools for improved use, research and delivery of digital cultural resources.

\section{References}

1. Agre, G., D. Dochev, L. Slavkova. Technology Enhanced Learning for Humanities by Active Learning. The SINUS Project Approach. - Cybernetics and Information Technologies, Vol. 12, 2012, No 4, pp. 25-42.

2. Arapi, P., D. Pan eva-Marin ova, R. Pavlov, S. Chris tod oulakis. Techniques to Personalized Observation and Improved Learning Experience in Digital Libraries. - In: Proc. of International Conference on e-Learning'16, Bratislava, Slovakia, 2016, pp. 94-100.

3. Arapi, P., N. Moumoutzis, M. Mylonakis, S. Christodoulakis. A PedagogyDriven Personalization Framework to Support Adaptive Learning Experiences. - In: Proc. of 7th IEEE International Conference on Advanced Learning Technologies (ICALT'07), Niigata, Japan, 2007, pp. 96-97.

4. ArchAIDE Project. Archaeological Automatic Interpretation and Documentation of Ceramics, Project Web Page, 2016, Retrieved 20 March 2017. http://www.archaide.eu/

5. ARCHESproject. Accessible Resources for Cultural Heritage EcoSystems, Project Web Page, 2016, Retrieved 20 May 2017. http://arches-project.eu/

6. B lo o m, B. S. Taxonomy of Educational Objectives: The Classification of Educational Goals, Book 1. Cognitive Domain. New York, Logman, USA, 1956.

7. CROSSCULTproject. Empowering Reuse of Digital Cultural Heritage in Context-Aware Crosscuts of European History, Project Web Site, 2016, Retrieved 20 May 2017. http://www.crosscult.eu/

8. CultEcoSys. Research Project No DN02/06/15.12.2016 - Concepts and Models for Innovation Ecosystems of Digital Cultural Assets, 2017, Retrieved 20 March 2017. http://cultecosys.math.bas.bg

9. ECHOES Project. Empowering Communities with a Heritage Open EcoSystem, Project Web Site, 2015, Retrieved 19 June 2017. https://www.erfgoedleiden.nl/images/dmdocuments/ECHOES\%20B\%201-3.pdf

10. European Commission. Implementation of Commission Recommendation on the Digitisation and Online Accessibility of Cultural Material and Digital Preservation, Progress Report 2011-2015, May 2016, Retrieved 15 June 2017.

http://ec.europa.eu/information_society/newsroom/image/document/2016-43/20132015_progress_report_18528.pdf

11. I-Media-Cities Project. Innovative e-Environment for Research on Cities and the Media, Project Web Page, 2016, Retrieved 20 March 2017. https://imediacities.eu/

12. ISO9241-1. Ergonomic Requirements for Office Work with Visual Display Terminals (VDTs): Part 11: Guidance on Usability, International Organization for Standardization, 1998.

13. Miles, M., A. Huberman, J. S aldana. Qualitative Data Analysis. 3th Ed. SAGE Publications Inc., California, USA, 2014.

14. M o r vill e, P. User Experience Design, 2004, Retrieved 20 March 2017. http://semanticstudios.com/user_experience_design/ 
15. P a n e v a, D. Ontology-Based Student Modeling. - In: Proc. of Open Workshop on Ubiquitous Learning Challenges: Design, Experiments and Context Aware Ubiquitous Learning, Turin, Italy, 2006, pp. 17-21.

16. Paneva-Marinova, D. A Semantic-Oriented Architecture of a Functional Module for Personalized and Adaptive Access to the Knowledge in a Multimedia Digital Library. Serdica Journal of Computing, Vol. 2, 2008, pp. 403-424.

17. Pan eva-M arinova, D., L. P a v lova-Drag an ova, L. Drag a nov, V. Georgi ev. Ontological Presentation of Analysis Method for Technology-Enhanced Learning. - In: Proc. of International Conference on Computer Systems and Technologies ComSysTech'12, Ruse, Bulgaria, 2012, pp. 384-390.

18. Pan eva-M a rinova, D., M. Goynov, D. Luchev, R. Pavlov. Solution for Content Interoperability among Digital Libraries for Orthodox Artefacts and Knowledge. - In: Proc. of 16th International Conference on Computer Systems and Technologies, Dublin, UK, 2015, pp. $168-175$.

19. Paneva-M arinova, D., M. Goynov, D. Luchev. Multimedia Digital Library: Constructive Block in Ecosystems for Digital Cultural Assets. Basic Functionality and Services. LAMBERT Academic Publishing, Germany, 2017.

20. Pan e va-Mari nova, D., M. Go ynov, R. P a v lov. Content Analyzing and Synthesizing Services in a Digital Library. - International Journal Information Technologies and Knowledge, Vol. 2, 2011, No 4, pp. 403-416.

21. P a n e va-M a ri n ov a, D., R. P a v lov. Educational Application of top of Digital Library for Cultural Heritage. - In: Proc. of International Conference on e-Learning and the Knowledge Society (E-Learning'11), Bucharest, Romania, 2011, pp. 211-224.

22. P a v lov, R., D. P a e va. Toward Ubiquitous Learning Application of Digital Libraries with Multimedia Content. - Cybernetics and Information Technologies, Vol. 6, 2006, No 3, pp. 51-62.

23. REFLECTIVE-6-2015. Innovation Ecosystems of Digital Cultural Assets, 2015, Retrieved 20 March 2017.

http://cordis.europa.eu/programme/rcn/664970_en.html

24. SINUS. Research Project No D-002-189 Semantic Technologies for Web Services and Technology Enhanced Learning, Project Web Page, Retrieved 20 March 2017.

http://sinus.iinf.bas.bg 